\title{
The anti-epithelial cell adhesion molecule (EpCAM) monoclonal antibody EpMab-16 exerts antitumor activity in a mouse model of colorectal adenocarcinoma
}

\author{
HIDEKI HOSONO $^{1 *}$, TOMOKAZU OHISHI ${ }^{2 *}$, JUNKO TAKEI $^{1 *}$, TEIZO ASANO $^{1}$, \\ YUSUKE SAYAMA $^{1}$, MANABU KAWADA ${ }^{2}$, MIKA K. KANEKO ${ }^{1}$ and YUKINARI KATO ${ }^{1,3}$ \\ ${ }^{1}$ Department of Antibody Drug Development, Tohoku University Graduate School of Medicine,
} Sendai, Miyagi $980-8575 ;{ }^{2}$ Institute of Microbial Chemistry (BIKAKEN), Numazu, Microbial Chemistry Research Foundation, Numazu, Shizuoka 410-0301; ${ }^{3}$ New Industry Creation Hatchery Center, Tohoku University, Sendai, Miyagi 980-8575, Japan

Received June 18, 2020; Accepted October 6, 2020

DOI: $10.3892 / \mathrm{ol} .2020 .12246$

\begin{abstract}
The epithelial cell adhesion molecule (EpCAM), which is a calcium-independent homophilic intercellular adhesion factor, contributes to cell signaling, differentiation, proliferation and migration. EpCAM is essential for carcinogenesis in numerous types of human cancer. The purpose of the present study was to establish an anti-EpCAM monoclonal antibody $(\mathrm{mAb})$ for targeting colorectal adenocarcinomas. Thus, an anti-EpCAM mAb, EpMab-16 $\left(\operatorname{IgG}_{2 \mathrm{a}}, \kappa\right)$, was established by immunizing mice with EpCAM-overexpressing CHO-K1 cells, and validated using flow cytometry, western blot, and immunohistochemical analyses. EpMab-16 reacted with endogenous EpCAM specifically in a colorectal adenocarcinoma cell line as determined by flow cytometry and western blot analyses. Immunohistochemical analysis demonstrated that EpMab-16 stained a plasma membrane-like pattern in clinical colorectal adenocarcinoma tissues. The dissociation constant $\left(K_{\mathrm{D}}\right)$ for EpMab-16 in a Caco-2 colorectal adenocarcinoma cell line determined by flow cytometry was $1.8 \times 10^{-8} \mathrm{M}$, suggesting moderate binding affinity of EpMab-16 for EpCAM. Whether the EpMab-16 induced antibody-dependent cellular cytotoxicity (ADCC) and complement-dependent cytotoxicity (CDC) against Caco-2 or antitumor activity was then assessed
\end{abstract}

Correspondence to: Professor Yukinari Kato, New Industry Creation Hatchery Center, Tohoku University, 2-1 Seiryo-machi, Aoba-ku, Sendai, Miyagi 980-8575, Japan

E-mail: yukinarikato@med.tohoku.ac.jp

*Contributed equally

Abbreviations: ADCC, antibody-dependent cellular cytotoxicity; CBIS, cell-based immunization and screening; CDC, complementdependent cytotoxicity; EpCAM, epithelial cell adhesion molecule; mAb, monoclonal antibody

Key words: EpCAM, monoclonal antibody, ADCC, CDC, antitumor activity, colorectal adenocarcinoma in a murine xenograft model. In vitro experiments revealed strong ADCC and CDC induction in Caco-2 cells by EpMab-16 treatment. In vivo experiments in a Caco-2 xenograft model demonstrated that EpMab-16 treatment significantly reduced tumor growth compared with that in mice treated with the control mouse IgG. These results suggested that EpMab-16 may be a promising treatment option for EpCAM-expressing colorectal adenocarcinomas.

\section{Introduction}

Cellular junctions comprise a range of cell adhesion molecules (CAM) and are essential for maintaining tissue architecture (1). The four major CAM families are integrins, cadherins, selectins and the immunoglobulin CAM superfamily (2). Integrins are composed of two or more noncovalently-associated membrane-spanning subunits $\alpha$ and $\beta$ (3). The specific combination of $\alpha$ and $\beta$ subunits confers specificity for various extracellular ligands and their respective intracellular signaling events, and each combination of $\alpha$ and $\beta$ represents a significant receptor family within the context of interaction with the extracellular matrix (3). Cadherins are calcium-dependent glycoproteins, which possess an extracellular CAM domain with three to five internal repeats, a single-span transmembrane domain and an intracellular domain (2). The extracellular domain of selectins consists of a calcium-dependent lectin domain and an epidermal growth factor (EGF)-like domain (2). Selectins also contain a hydrophobic transmembrane domain and a short cytoplasmic tail (2). The Ig-CAMs are calcium-independent, with an extracellular domain comprising a ligand-binding region of four to six Ig-like repeats, one to five fibronectin-like repeats, a transmembrane domain and an intracellular component (1). Although these families are predominant, a number of CAMs do not share any structural similarities with them, such as the epithelial cell adhesion molecule (EpCAM) (4).

EpCAM is one of the first identified human tumor-associated biomarkers (5) and is now considered to be a marker of tumor-initiating cells (6). EpCAM is a transmembrane, calcium-independent, homophilic, intercellular adhesion glyco- 
protein (314 amino acids; $40 \mathrm{kDa}$ ) with three distinct domains: An extracellular domain (EpEX, 265 amino acids), a transmembrane domain and an intracellular domain (EpICD, 26 amino acids) (7). The cleaved EpICD enters the nucleus, leading to the activation of the $\beta$-catenin/c-Myc signaling pathway to promote cancer cell proliferation (8). EpCAM functions include cell signaling, differentiation and migration in addition to adhesion and proliferation (4). EpCAM has been implicated in carcinogenesis and is expressed robustly in various types of human epithelial cancers, such as lung, breast, ovarian, cervical and colorectal cancer (CRC), suggesting that it may be a promising target for cancer diagnosis and therapy (9-11).

According to GLOBOCAN 2018 data, CRC is the third most commonly occurring cancer and second leading cause of cancer-associated death in the world, with $\sim 881,000$ deaths estimated for 2018 (12). Although surgical removal of cancer followed by adjuvant therapy is one of the most effective treatments, recurrences are inevitable (13-15). Antibody-based treatments are also currently used in patients with advanced CRC; however, the prognosis and clinical outcomes of patients with CRC remain poor (16). Therefore, new strategies are required to improve the effectiveness of CRC treatment.

The present study developed an anti-EpCAM monoclonal antibody $(\mathrm{mAb})$ using cell-based immunization and screening (CBIS) methods (17) aiming to determine whether these anti-EpCAM mAbs induced antibody-dependent cellular cytotoxicity (ADCC), complement-dependent cytotoxicity (CDC) or antitumor activity against CRC in a murine xenograft model.

\section{Materials and methods}

Antibodies. Purified mouse IgG (cat. no. I8765) and mouse $\mathrm{IgG}_{2 \mathrm{a}}$ (cat. no. M7769) were purchased from Sigma-Aldrich; Merck KGaA. Anti-EpCAM mAbs were purified using Protein G-Sepharose (Cytiva).

Animals. All animal experiments were performed in accordance with institutional guidelines and regulations to minimize animal suffering and distress in the laboratory. The Institutional Committee for Experiments of the Institute of Microbial Chemistry (Numazu, Japan) approved the animal studies for ADCC and antitumor activity (approval no. 2019-066). Mice were monitored for health and weight every 1-5 days. Experiments on mice were conducted in $\leq 3$ weeks. Weight loss $>25 \%$ or tumor size $>3,000 \mathrm{~mm}^{3}$ were identified as humane endpoints for euthanasia. At humane and experimental endpoints, mice were euthanized by cervical dislocation, and death was verified by validating respiratory and cardiac arrest.

Cell lines. P3X63Ag8U.1 (P3U1), CHO-K1 and Caco-2 cells were obtained from the American Type Culture Collection. The Genome Network Project clone IRAK021G03 (EpCAM) was provided by the RIKEN BioResource Research Center through the National BioResource Project of the MEXT and AMED agencies of Japan (18-21). EpCAM DNA plus a C-terminal PA tag recognized by the anti-PA tag mAb (NZ-1) was subcloned into a pCAG-Ble vector (FUJIFILM Wako Pure Chemical Corporation). CHO/EpCAM was established by transfecting pCAG/EpCAM-PA into $\mathrm{CHO}-\mathrm{K} 1$ cells using the Neon Transfection System (Thermo Fisher Scientific, Inc.). CHO-K1 cells $\left(1.5 \times 10^{6}\right)$ were transfected with $10 \mu \mathrm{g}$ of plasmid DNA using $100 \mu \mathrm{l}$ Neon tip, at room temperature. After 4 days, cells were incubated with $1 \mu \mathrm{g} / \mathrm{ml}$ anti-EpCAM mAb (clone 9C4; cat. no. 324202; BioLegend, Inc.) for $30 \mathrm{~min}$ on ice and subsequently with Alexa Fluor 488-conjugated anti-mouse IgG (1:1,000; cat. no. 4408; Cell Signaling Technology, Inc.) for $30 \mathrm{~min}$ on ice. Positive cells for anti-EpCAM mAb were sorted using an SH800 cell sorter (Sony Corporation), and stable transfectants were cultivated in RPMI-1640 medium (Nacalai Tesque, Inc.) containing $0.5 \mathrm{mg} / \mathrm{ml}$ zeocin (InvivoGen). Using TruGuide gRNA tool, gRNA of EpCAM (NM_002354) was selected from GeneArt predesigned gRNAs database (Thermo Fisher Scientific, Inc.). gRNA sequence used was GATCCTGACTGCGATGAGAG(cgg), which targeted exon 3 of EpCAM (Assay ID, CRISPR701274). Double strand gRNA sequence was subcloned into GeneArt CRISPR Nuclease Vector with OFP Reporter (Thermo Fisher Scientific, Inc.). Caco-2/EpCAM-knockout (BINDS-16) cells were generated by transfecting $10 \mu \mathrm{g}$ of CRISPR/Cas9 plasmids for EpCAM (Thermo Fisher Scientific, Inc.) into Caco-2 cells $\left(1.5 \times 10^{6}\right)$ for 7 days using a Neon transfection system with $100 \mu 1$ Neon tip. Stable transfectants were established by cell sorting as aforementioned. P3U1, CHO-K1 and CHO/EpCAM cells were cultured in RPMI-1640 medium (Nacalai Tesque, Inc.). Caco-2 and BINDS-16 were cultured in Dulbecco's modified Eagle's medium (DMEM; Nacalai Tesque, Inc.). The medium was supplemented with $10 \%$ heat-inactivated fetal bovine serum (FBS; Thermo Fisher Scientific Inc.), $100 \mathrm{U} / \mathrm{ml}$ penicillin, $100 \mu \mathrm{g} / \mathrm{ml}$ streptomycin and $0.25 \mu \mathrm{g} / \mathrm{ml}$ amphotericin $\mathrm{B}$ (Nacalai Tesque, Inc.), and the cells were incubated at $37^{\circ} \mathrm{C}$ in a humidified atmosphere containing $5 \% \mathrm{CO}_{2}$.

Hybridoma production. The CBIS method (17) was used in the present study to develop mAbs against EpCAM. Briefly, one $\mathrm{BALB} / \mathrm{c}$ mouse was intraperitoneally (i.p.) immunized with $\mathrm{CHO} / \mathrm{EpCAM}$ cells $\left(1 \times 10^{8}\right.$ cells $\left./ 500 \mu \mathrm{l}\right)$ with Imject Alum adjuvant (Thermo Fisher Scientific Inc.). The procedure included three additional immunizations, followed by a final booster injection administered i.p. 2 days before spleen cell harvesting. Spleen cells $\left(1 \times 10^{8}\right.$ cells) were then fused with mouse plasma cell myeloma P3U1 cells $\left(1 \times 10^{7}\right.$ cells $)$ using PEG1500 (Roche Diagnostics). The hybridomas were cultured in RPMI-1640 medium supplemented with hypoxanthine, aminopterin and thymidine for selection (50X solution; Thermo Fisher Scientific Inc.). Cell culture supernatants of hybridomas in each well of 96-well plates were mixed with $1 \times 10^{5} \mathrm{CHO} / \mathrm{EpCAM}$ cells and were directly screened using flow cytometry.

Flow cytometry. Caco-2 cells $\left(2.5 \times 10^{5}\right.$ cells $\left./ \mathrm{ml}\right)$ were harvested after brief exposure to $0.25 \%$ trypsin in $1 \mathrm{mM}$ ethylenediaminetetraacetic acid (EDTA; Nacalai Tesque, Inc.). Following washing with $0.1 \%$ bovine serum albumin (BSA; Nacalai Tesque, Inc.) in phosphate-buffered saline (PBS), Caco- 2 cells were treated with $1 \mu \mathrm{g} / \mathrm{ml}$ of anti-EpCAM $\mathrm{mAbs}$ for $30 \mathrm{~min}$ at $4^{\circ} \mathrm{C}$, followed by Alexa Fluor 488-conjugated anti-mouse IgG (1:1,000; cat. no. 4408; Cell Signaling Technology, Inc.). Fluorescence data were obtained using an EC800 Cell Analyzer (Sony Corporation), and analyzed 
using the originally installed EC800 software v1.3.6 (Sony Corporation).

Western blot analysis. Cell pellets were lysed in PBS with $1 \%$ Triton X-100 and $50 \mu \mathrm{g} / \mathrm{ml}$ aprotinin (cat. no. 03346-84; Nacalai Tesque, Inc.). Protein concentration was determined using the BCA assay. Cell lysates of CHO-K1, CHO/EpCAM, Caco-2 and BINDS-16 cells were boiled in sodium dodecyl sulfate sample buffer (Nacalai Tesque, Inc.). The samples (10 $\mu \mathrm{g} / \mathrm{lane})$ were then electrophoresed on $5-20 \%$ polyacrylamide gels (Nacalai Tesque, Inc.) and transferred to polyvinylidene difluoride membranes (Merck KGaA). Following blocking with $4 \%$ milk (Nacalai Tesque, Inc.) for $1 \mathrm{~h}$, the membrane was incubated with anti-EpCAM $(1 \mu \mathrm{g} / \mathrm{ml})$ or anti- $\beta$-actin $(1 \mu \mathrm{g} / \mathrm{ml}$; clone AC- 15 ; cat no. A5441; Sigma-Aldrich; Merck KGaA) antibodies for $1 \mathrm{~h}$, followed by incubation with HRP-conjugated anti-mouse IgG (cat. no. P0260, Agilent Technologies, Inc.) or anti-rat IgG (cat. no. A9542; Sigma-Aldrich; Merck KGaA) at a 1:2,000 dilution for $1 \mathrm{~h}$ at room temperature. The membrane was developed using the ImmunoStar LD Chemiluminescence Reagent (FUJIFILM Wako Pure Chemical Corporation) and a Sayaca-Imager (DRC Co., Ltd.). All western blotting procedures were performed at room temperature.

Immunohistochemical analyses. Histological sections $(4 \mu \mathrm{m})$ of a colorectal adenocarcinoma tissue array (cat. no. CO243b; US Biomax Inc.) were autoclaved directly in citrate buffer (pH 6.0; Nichirei Bioscience, Inc.) for 20 min. The sections were then incubated with $1 \mu \mathrm{g} / \mathrm{ml}$ anti-EpCAM $\mathrm{mAb}$ for $1 \mathrm{~h}$ at room temperature and treated using an Envision+ kit (Agilent Technologies, Inc.) for $30 \mathrm{~min}$ at room temperature. The color was developed using 3,3'-diaminobenzidine tetrahydrochloride (Agilent Technologies Inc.) for $2 \mathrm{~min}$ at room temperature, and sections were then counterstained with hematoxylin (FUJIFILM Wako Pure Chemical Corporation) for $2 \mathrm{~min}$ at room temperature. Hematoxylin and eosin (H\&E) staining (FUJIFILM Wako Pure Chemical Corporation) was performed using consecutive colorectal adenocarcinoma tissue sections for $2 \mathrm{~min}$ at room temperature.

Determination of the binding affinity. Caco-2 cells were suspended in $100 \mu \mathrm{l}$ serially diluted anti-EpCAM mAb $(0.006-100 \mu \mathrm{g} / \mathrm{ml})$, followed by the addition of Alexa Fluor 488-conjugated anti-mouse IgG (1:200; cat. no. 4408; Cell Signaling Technology, Inc.). Fluorescence data were obtained using an EC800 Cell Analyzer, and analyzed using the originally installed EC800 v1.3.6 software (Sony Corporation). The dissociation constant $\left(K_{\mathrm{D}}\right)$ was calculated by fitting binding isotherms to built-in, one-site binding models in GraphPad Prism 6 (GraphPad Software, Inc.).

ADCC. ADCC induction by EpCAM was assayed as follows: A total of 6 female 5-week-old BALB/c nude mice were purchased from Charles River Laboratories, Inc. Following euthanasia by cervical dislocation, the spleens were removed aseptically, and single-cell suspensions were obtained by forcing spleen tissues through a sterile cell strainer (cat. no. 352360; Corning, Inc.) with a syringe. Erythrocytes were lysed by 10 -sec exposure to ice-cold distilled water. The splenocytes were washed with DMEM and resuspended in DMEM with 10\% FBS; this yield was designated as effector cells. Caco-2 cells were labeled with $10 \mu \mathrm{g} / \mathrm{ml}$ Calcein-AM (Thermo Fisher Scientific, Inc.) and resuspended in DMEM with $10 \%$ FBS. Caco-2 cells were transferred to 96-well plates at $2 \times 10^{4}$ cells/well and mixed with the effector cells at an effector-to-target ratio of 50:1, along with $100 \mu \mathrm{g} / \mathrm{ml}$ anti-EpCAM mAb or control mouse $\operatorname{IgG}_{2 \mathrm{a}}$. Following a 4-hincubation, Calcein-AM release into the supernatant was measured in each well using a Power Scan HT microplate reader (BioTek Instruments, Inc.) with an excitation wavelength of $485 \mathrm{~nm}$ and an emission wavelength of $538 \mathrm{~nm}$. Cytolytic activity was determined as a percentage of lysis and calculated using the following equation: Lysis $(\%)=(\mathrm{E}-\mathrm{S}) /(\mathrm{M}-\mathrm{S}) \times 100$, where $\mathrm{E}$ is the fluorescence measured in the co-cultures of target and effector cells, $\mathrm{S}$ is the spontaneous fluorescence of the target cells, and $\mathrm{M}$ is the maximum fluorescence measured after lysis of all cells with a buffer containing $0.5 \%$ Triton X-100, $10 \mathrm{mM}$ Tris- $\mathrm{HCl}(\mathrm{pH}$ 7.4) and $10 \mathrm{mM}$ EDTA.

CDC. CDC by EpCAM was assayed as follows: Caco-2 cells were labeled with $10 \mu \mathrm{g} / \mathrm{ml}$ Calcein-AM and resuspended in DMEM with $10 \%$ FBS. Caco-2 cells were plated in 96-well plates at $2 \times 10^{4}$ cells/well, and $10 \%$ Low-Tox-M rabbit complement (Cedarlane Laboratories) with $100 \mu \mathrm{g} / \mathrm{ml}$ anti-EpCAM $\mathrm{mAb}$ or control mouse $\operatorname{IgG}_{2 \mathrm{a}}$ was added to each well. Following a 4-h incubation at $37^{\circ} \mathrm{C}$, Calcein-AM release into the supernatant was determined in each well. Fluorescence intensity was calculated as described in the ADCC section.

Antitumor activity of anti-EpCAM $m A b$ in colorectal adenocarcinoma xenografts. A total of 325 -week-old female BALB/c nude mice were purchased from Charles River Laboratories, Inc. After a 2-week acclimation period, the mice were used in experiments at 7 weeks of age. Caco- 2 cells $\left(0.3 \mathrm{ml} ; 1.33 \times 10^{8}\right.$ cells $/ \mathrm{ml}$ in DMEM) were mixed with $0.5 \mathrm{ml}$ BD Matrigel Matrix Growth Factor Reduced (BD Biosciences), and $100 \mu \mathrm{l}$ of this suspension $\left(5 \times 10^{6}\right.$ cells) was injected subcutaneously into the left flank of each animal. On day 1 post-inoculation, $100 \mu \mathrm{g}$ anti-EpCAM $\mathrm{mAb}$ or control mouse IgG in $100 \mu \mathrm{l}$ PBS was injected i.p. Additional antibody inoculations were performed on days 7 and 12. Tumor formation was measured in mice in the treatment and control groups on days 7, 8, 12,15 and 17 after Caco-2 cell injection. On day 17 after cell implantation, all mice were euthanized by cervical dislocation, and tumor diameters and volumes were measured and recorded.

Statistical analyses. Data are presented as the mean \pm SEM. Statistical analysis was conducted with one-way ANOVA and Tukey's multiple comparisons tests for ADCC and CDC; one-way ANOVA and Sidak's multiple comparisons tests for tumor volume and mouse weight; and Welch's t-test for tumor weight. All calculations were performed with GraphPad Prism 7 (GraphPad Software, Inc.). P $<0.05$ was considered to indicate a statistically significant difference.

\section{Results}

Establishment and characterization of the anti-EpCAM $m A b$. The anti-EpCAM mAb was established by immunizing one 
A

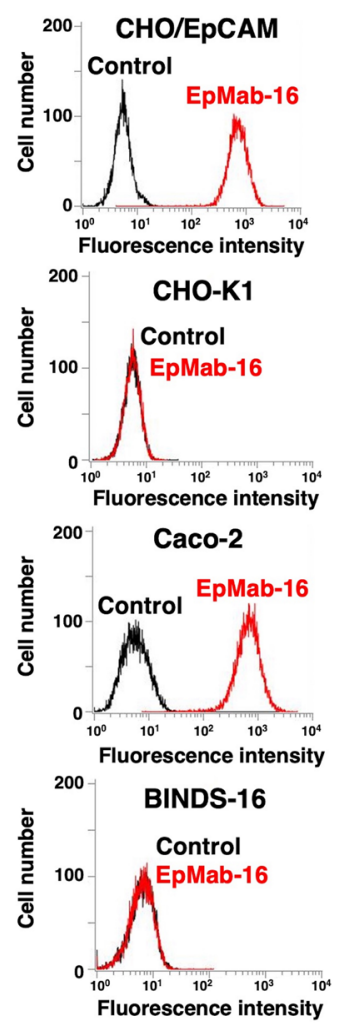

B

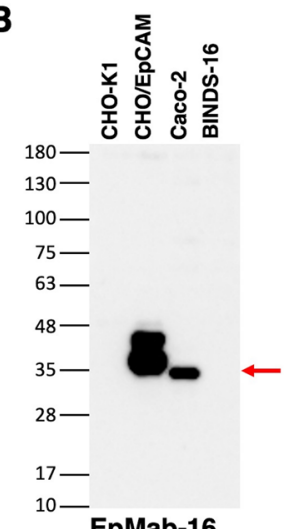

EpMab-16

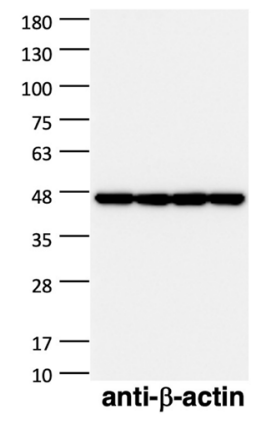

Figure 1. Recognition of EpCAM by EpMab-16. (A) Flow cytometry analysis using EpMab-16. CHO/EpCAM, CHO-K1, Caco-2 and BINDS-16 cells were treated with EpMab-16 $(1 \mu \mathrm{g} / \mathrm{ml})$ or buffer control, followed by secondary antibodies. (B) Western blot analysis of EpCAM expression using EpMab-16. Red arrow denotes 35-kDa EpCAM. EpCAM, epithelial cell adhesion molecule; EpMab-16, anti-EpCAM monoclonal antibody; BINDS-16, Caco-2/EpCAM-knockout.

mouse with $\mathrm{CHO} / \mathrm{EpCAM}$ cells and fusing its spleen cells with P3U1 cells. Supernatants from hybridomas, which were positive for $\mathrm{CHO} / \mathrm{EpCAM}$ and negative for $\mathrm{CHO}-\mathrm{K} 1$, were selected by flow cytometry. Further screening by immunohistochemistry and western blotting was performed for validation, resulting in the establishment of EpMab-16 $\left(\operatorname{IgG}_{2 \mathrm{a}}, \kappa\right)$.

Flow cytometry was performed to assess the sensitivity of EpMab-16 in CHO/EpCAM cells and the Caco-2 colorectal adenocarcinoma cell line. As presented in Fig. 1A, EpMab-16 bound to $\mathrm{CHO} / \mathrm{EpCAM}$ cells, but not $\mathrm{CHO}-\mathrm{K} 1$ cells. EpMab-16 also bound to Caco-2 but not BINDS-16 cells, indicating that EpMab-16 was specific for EpCAM in the colorectal adenocarcinoma cell line.

Western blotting was performed to further assess the sensitivity of EpMab-16. Lysates of CHO-K1, CHO/EpCAM, Caco-2 and BINDS-16 cells were probed. As demonstrated in Fig. 1B, EpMab-16 detected the 35-kDa band of EpCAM in lysates from $\mathrm{CHO} / \mathrm{EpCAM}$ and Caco-2 cells, whereas this band was not present in lysates from CHO-K1 and BINDS-16 cells, indicating that EpMab-16 specifically detected both exogenous and endogenous EpCAM. The molecular weight of EpCAM between $\mathrm{CHO} / \mathrm{EpCAM}$ and Caco-2 was different as a PA tag (12 amino acids) was added to $\mathrm{C}$-terminus of EpCAM in $\mathrm{CHO} / \mathrm{EpCAM}$ cells.

EpMab-16 detected membrane antigens in colorectal adenocarcinoma tissues in the immunohistochemical analysis (Fig. 2A and B). Among six cases of colorectal adenocarci-

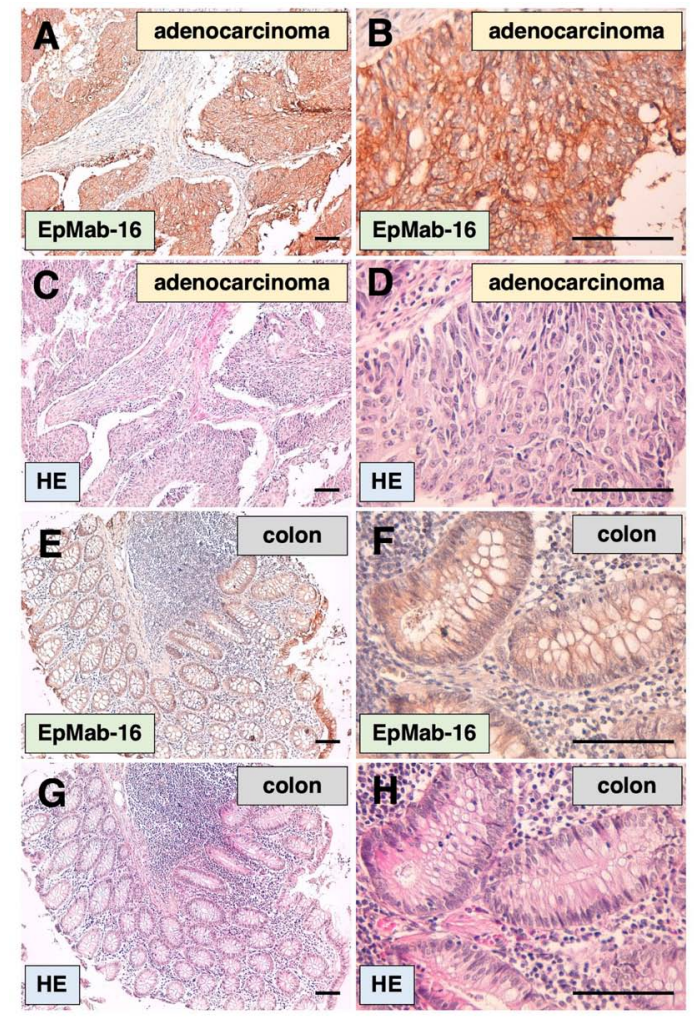

Figure 2. Immunohistochemical analysis of colorectal adenocarcinoma and normal colon tissues using EpMab-16. (A and B) FFPE tissue sections of colorectal adenocarcinoma stained with $1 \mu \mathrm{g} / \mathrm{ml}$ EpMab-16. B is a magnified version of A. (C and D) HE staining of consecutive colorectal adenocarcinoma tissue sections. D is a magnified version of C. (E and F) FFPE tissue sections of normal colon stained with $1 \mu \mathrm{g} / \mathrm{ml}$ EpMab-16. F is a magnified version of $\mathrm{E}$. ( $\mathrm{G}$ and $\mathrm{H}$ ) $\mathrm{HE}$ staining against consecutive normal colon tissue sections. $\mathrm{H}$ is a magnified version of G. Scale bar, $100 \mu \mathrm{m}$. EpMab-16, antiepithelial cell adhesion molecule monoclonal antibody; FFPE, formalin-fixed paraffin-embedded; HE, hematoxylin and eosin.

noma, five ( $83 \%$ ) were positively stained by EpMab-16. No staining was observed without the primary $\mathrm{mAb}$ (data not shown). H\&E staining was performed to stain nucleus as blue and cytosol as pink, using consecutive colorectal adenocarcinoma tissue sections (Fig. 2C and D). Furthermore, EpMab-16 weakly detected membrane antigens in normal colon tissues (Fig. 2E and F). Among six samples of normal colon tissues, three tissues (50\%) were stained by EpMab-16. No staining was observed without the primary mAb (data not shown). $\mathrm{H} \& \mathrm{E}$ staining was performed to stain nucleus as blue and cytosol as pink, using consecutive normal colon tissue sections (Fig. 2G and H).

Kinetic analysis of the interactions of EpMab-16 with Caco- 2 cells was subsequently analyzed using flow cytometry. The $K_{\mathrm{D}}$ for EpMab-16 in Caco-2 cells was calculated to be $1.8 \times 10^{-8} \mathrm{M}$ (Fig. 3A), indicating a moderate binding affinity of EpMab-16 to colorectal adenocarcinoma cells.

ADCC and CDC activities of EpMab-16 in a colorectal adenocarcinoma cell line. The present study further investigated whether EpMab-16 induced ADCC and CDC antitumor activity in an EpCAM-expressing Caco-2 colorectal adenocarcinoma cell line. As presented in Fig. 3B, EpMab-16 exhibited higher ADCC (44\% cytotoxicity) in Caco- 2 cells compared 
A

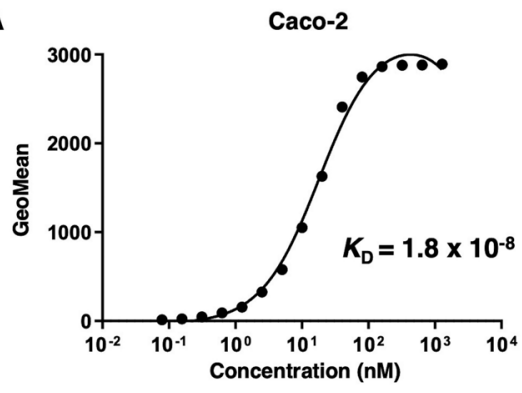

B

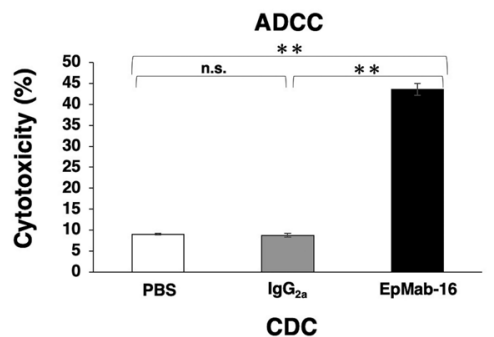

C

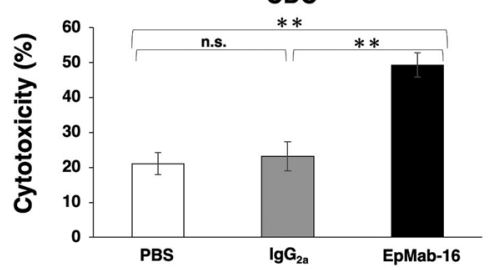

Figure 3. Binding affinity, ADCC and CDC of EpMab-16. (A) Determination of binding affinity of EpMab-16 in Caco-2 cells. (B) ADCC activity of EpMab-16, control mouse $\mathrm{IgG}_{2 \mathrm{a}}$ and control PBS in Caco-2 cells. (C) CDC activity of EpMab-16, control mouse $\mathrm{IgG}_{2 \mathrm{a}}$ and control PBS in Caco-2 cells. ${ }^{* *} \mathrm{P}<0.01$; n.s., not significant. EpMab-16, anti-epithelial cell adhesion molecule monoclonal antibody; ADCC, antibody-dependent cellular cytotoxicity; CDC, complement-dependent cytotoxicity; $K_{\mathrm{D}}$, dissociation constant.

with that of control mouse $\operatorname{IgG}_{2 \mathrm{a}}(8.7 \%$ cytotoxicity; $\mathrm{P}<0.01)$ or control PBS $(9.0 \%$ cytotoxicity; $\mathrm{P}<0.01)$ treatment. EpMab-16 was also associated with more robust CDC activity ( $49 \%$ cytotoxicity) in Caco-2 cells compared with that of control mouse $\operatorname{IgG}_{2 \mathrm{a}}(23 \%$ cytotoxicity; $\mathrm{P}<0.01)$ or control PBS $(21 \%$ cytotoxicity; $\mathrm{P}<0.01$ ) treatment (Fig. 3C). These results suggested that EpMab-16 induced strong ADCC and CDC antitumor activity in vitro.

EpMab-16 antitumor activity in mouse xenografts of Caco-2 colorectal adenocarcinoma cells. We further examined whether EMab-16 exerts antitumor activity in vivo. On days 1, 7 and 12 after Caco-2 cell injections into the mice, the Caco-2 xenograft mouse models were injected with EpMab-16 or control mouse IgG. During the animal experiment, no apparent weight loss due to tumor burden or organ failure was observed among the mice. EpMab-16-treated mice exhibited significantly lower tumor growth on days 7,8 , 12,15 and 17 (all $\mathrm{P}<0.01$ ) compared with that in IgG-treated control mice (Fig. 4A). Tumor volume reduction by EpMab-16 treatment reached $66 \%$ relative to the control group on day 17. Tumors from EpMab-16-treated mice weighed significantly less compared with those from IgG-treated control mice $(60 \%$ reduction; $\mathrm{P}<0.01$; Fig. $4 \mathrm{~B})$. Resected tumors on day 17 are depicted in Fig. 4C. Total body weights did not significantly differ between the treatment and control groups (Fig. S1). These results indicated that EpMab-16 reduced the
A

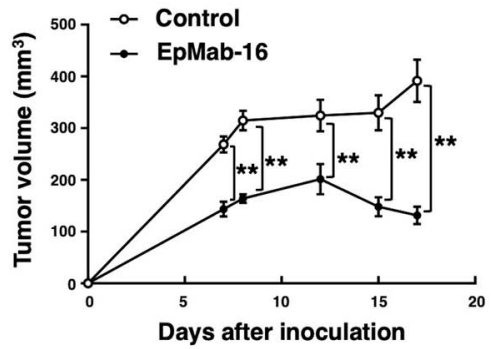

B

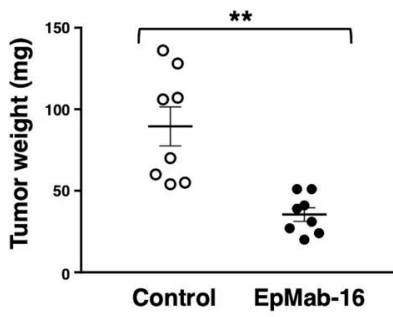

C

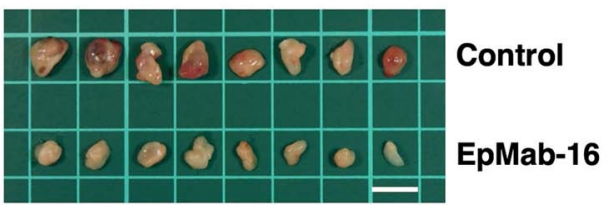

Figure 4. Antitumor activity of EpMab-16 in Caco-2 xenografts. (A) Tumor volumes of Caco-2 xenografts. Caco-2 cells were injected subcutaneously, and $100 \mu \mathrm{g}$ EpMab-16 or control mouse IgG was injected into the treatment and control mice, respectively; additional antibodies were injected on days 7 and 12. Tumor volume was measured on days 7, 8, 12, 15 and 17 . (B) Tumor weights of Caco-2 xenografts resected from the EpMab-16 and control mouse IgG groups on day 17. (C) Resected tumors of Caco-2 xenografts from the EpMab-16 and control mouse IgG groups on day 17. Scale bar, $1 \mathrm{~cm} .{ }^{* *} \mathrm{P}<0.01$. EpMab-16, anti-epithelial cell adhesion molecule monoclonal antibody.

growth of Caco-2 xenografts, but did not altogether eliminate it.

\section{Discussion}

The present study aimed to determine whether a novel anti-EpCAM mAb may be useful for treating colorectal adenocarcinoma. First, a sensitive and specific anti-EpCAM mAb, EpMab-16, was developed, which exhibited high reactivity for colorectal adenocarcinoma by flow cytometry, western blotting and immunohistochemical analyses. Notably, the results also suggested that EpMab-16 exhibited diagnostic efficacy in FFPE tissues as pathological diagnosis utilizes FFPE tissues. EpMab-16 was demonstrated to possess strong ADCC and CDC activity against the Caco-2 colorectal adenocarcinoma cell line in vitro, and significantly reduced not only the volume but also the weight of Caco-2 xenografts in vivo. Although tumor reduction was determined to be $66 \%$ by volume and $60 \%$ by weight on day 17 , the reduction was not sufficient to eliminate the tumor entirely.

Cancer stem cells (CSCs) exhibit specific characteristics including decontrolled self-renewal, tumor-initiating and tumor-promoting properties, and chemotherapy resistance. Therefore, targeting CSCs is thought to be a promising approach to treat cancer (22). Since CD133 is thought to be 
one of CSC markers for CRC (23-25), we previously developed an anti-CD133 mAb (CMab-43, mouse $\mathrm{IgG}_{2 \mathrm{a}}$ ) (17) and investigated whether CD133 may represent a therapeutic target in colorectal adenocarcinoma using CMab-43 (26). Importantly, CMab-43 exerted antitumor activity in Caco-2 xenograft models at a dose of $100 \mu \mathrm{g} /$ mouse/week administered three times, suggesting that CMab-43 may be useful for antibody therapy against CD133-positive CRC (26). In the present study, the effects of an anti-EpCAM mAb, EpMab-16, were examined in vitro and in vivo, as EpCAM is also known to be a CSC marker for CRC (25). In the present study, EpMab-16 also showed antitumor activity against CRC in a murine xenograft model. CSC marker-expressing cells may be heterogenous; therefore, the combination of mAbs or anticancer drugs to kill the CSC marker-expressing cancer cells could be an ideal strategy to treat CRC.

In another recent study, we established an anti-HER2 mAb $\left(\mathrm{H}_{2}\right.$ Mab-19, mouse $\left.\mathrm{IgG}_{2 \mathrm{~b}}\right)$ (27). Our previous study demonstrated that $\mathrm{H}_{2} \mathrm{Mab}-19$ significantly reduced tumor development in HER2-expressing breast cancer cells (BT-474), oral cancer cells (HSC-2 and SAS) and CRC cells (Caco-2) xenograft models, suggesting that treatment with $\mathrm{H}_{2} \mathrm{Mab}-19$ may be a useful therapy for patients with HER2-expressing cancers $(27,28)$. Although $\mathrm{H}_{2}$ Mab-19 showed significant antitumor activity, immunohistochemical analysis revealed that HER2 expression was diminished in the remaining cancer cells after $\mathrm{H}_{2} \mathrm{Mab}-19$ treatment (28). These results suggest that $\mathrm{H}_{2} \mathrm{Mab}-19$ treatment may not be fully effective for patients with HER2-expressing cancers. In the current study, an EpCAM-targeting mAb, EpMab-16, was developed. To enhance the therapeutic effects in vivo, the combinational use of different mAbs, such as $\mathrm{H}_{2} \mathrm{Mab}-19$ and EpMab-16 may be required to treat HER2 and/or EpCAM-expressing CRC cells.

The success of antibody-based therapeutics of cancer depends on the target antigen and the antibody to be used. It has been reported that EpCAM is upregulated in $94 \%$ of CRCs (29), whereas EpCAM expression is restricted to only the basolateral membrane of epithelial cells in normal tissues, and therapeutic agents have limited access to it (30), underscoring the importance of EpCAM as a therapeutic target. Since EpMab-16 appears to react with not only the basolateral membrane, but also the apical membrane of epithelial cells according to the results of the present study, the expression pattern of EpCAM should be further investigated by comparing EpMab-16 with other anti-EpCAM mAbs.

A number of antibody-based therapeutic approaches targeting EpCAM have been developed (10). Previously, Liao et al (31) generated an anti-EpCAM mAb EpAb2-6. Using a colon cancer xenograft model, they demonstrated that EpAb2-6 induced CRC cell death by inhibiting EpCAM signaling rather than by acting through the ADCC or CDC (31). On the other hand, EpMab-16 generated in the present study possessed high ADCC and CDC activities against CRC cells and exhibited antitumor activity in vivo. Another previous study demonstrated that an anti-EpCAM toxin-conjugated antibody Oportuzumab monatox (also termed VB4-845) was effective against squamous cell carcinomas of the head and neck and non-muscle invasive bladder cancer, and well-tolerated in clinical trials (phase I and II) (32). Considering that EpCAM is also expressed in tumor-initiating cells, antibody-based thera- pies against EpCAM may kill not only proliferating cancer cells, but also drug-resistant dormant CRC cells (6). These results suggest that the EpCAM-targeted immunotherapy may be a promising therapeutic strategy for CRC. To further develop EpCAM-targeted cancer therapy, further studies are required to elucidate the precise role of EpCAM inhibition among various cancer cells.

\section{Acknowledgements}

The authors would like to thank Mr. Takuro Nakamura, Ms. Miyuki Yanaka, Ms. Saori Handa, Ms. Saki Okamoto and Mr. Yu Komatsu (Department of Antibody Drug Development, Tohoku University Graduate School of Medicine, Sendai, Japan) for technical assistance with the in vitro experiments, and Ms. Akiko Harakawa [Institute of Microbial Chemistry (BIKAKEN), Microbial Chemistry Research Foundation, Numazu, Japan] for technical assistance with the animal experiments.

\section{Funding}

This research was supported in part by the Japan Agency for Medical Research and Development (grant nos. JP20am0401013 to YK, JP20am0101078 to YK and JP20ae0101028 to YK).

\section{Availability of data and materials}

The datasets used and/or analyzed during the study are available from the corresponding author on reasonable request.

\section{Authors' contributions}

$\mathrm{HH}, \mathrm{TA}, \mathrm{YS}$, and MKK performed the in vitro experiments. TO performed in vivo experiments. JT analyzed the experimental data. MK and YK designed the study and wrote the manuscript. All authors read and approved the final manuscript.

\section{Ethics approval and consent to participate}

Animal studies for ADCC and the antitumor activity were approved by the Institutional Committee for Experiments of the Institute of Microbial Chemistry (Numazu, Japan; approval no. 2019-066).

\section{Patient consent for publication}

Not applicable.

\section{Competing interests}

The authors declare that they have no competing interests.

\section{References}

1. Okegawa T, Pong RC, Li Y and Hsieh JT: The role of cell adhesion molecule in cancer progression and its application in cancer therapy. Acta Biochim Pol 51: 445-457, 2004.

2. Petruzzelli L, Takami M and Humes HD: Structure and function of cell adhesion molecules. Am J Med 106: 467-476, 1999. 
3. Maheshwari G, Brown G, Lauffenburger DA, Wells A and Griffith LG: Cell adhesion and motility depend on nanoscale RGD clustering. J Cell Sci 113: 1677-1686, 2000.

4. Trzpis M, McLaughlin PM, de Leij LM and Harmsen MC: Epithelial cell adhesion molecule: More than a carcinoma marker and adhesion molecule. Am J Pathol 171: 386-395, 2007.

5. Herlyn M, Steplewski Z, Herlyn D and Koprowski H: Colorectal carcinoma-specific antigen: Detection by means of monoclonal antibodies. Proc Natl Acad Sci USA 76: 1438-1442, 1979.

6. Imrich S, Hachmeister M and Gires O: EpCAM and its potential role in tumor-initiating cells. Cell Adhes Migr 6: 30-38, 2012.

7. Litvinov SV, Velders MP, Bakker HA, Fleuren GJ and Warnaar SO: Ep-CAM: A human epithelial antigen is a homophilic cell-cell adhesion molecule. J Cell Biol 125: 437-446, 1994

8. Maetzel D, Denzel S, Mack B, Canis M, Went P, Benk M, Kieu C, Papior P, Baeuerle PA, Munz M, et al: Nuclear signalling by tumour-associated antigen EpCAM. Nat Cell Biol 11: 162-171, 2009.

9. Sen S and Carnelio S: Expression of epithelial cell adhesion molecule (EpCAM) in oral squamous cell carcinoma. Histopathology 68: 897-904, 2016.

10. Baeuerle PA and Gires O: EpCAM (CD326) finding its role in cancer. Br J Cancer 96: 417-423, 2007.

11. Went P, Vasei M, Bubendorf L, Terracciano L, Tornillo L, Riede U, Kononen J, Simon R, Sauter G and Baeuerle PA: Frequent high-level expression of the immunotherapeutic target Ep-CAM in colon, stomach, prostate and lung cancers. Br J Cancer 94: 128-135, 2006.

12. Bray F, Ferlay J, Soerjomataram I, Siegel RL, Torre LA and Jemal A: Global cancer statistics 2018: GLOBOCAN estimates of incidence and mortality worldwide for 36 cancers in 185 countries. CA Cancer J Clin 68: 394-424, 2018.

13. Wolpin BM, Meyerhardt JA, Mamon HJ and Mayer RJ: Adjuvant treatment of colorectal cancer. CA Cancer J Clin 57: 168-185, 2007.

14. Longo WE and Johnson FE: The preoperative assessment and postoperative surveillance of patients with colon and rectal cancer. Surg Clin North Am 82: 1091-1108, 2002.

15. O'Connell MJ, Colangelo LH, Beart RW, Petrelli NJ, Allegra CJ, Sharif S, Pitot HC, Shields AF, Landry JC, Ryan DP, et al: Capecitabine and oxaliplatin in the preoperative multimodality treatment of rectal cancer: Surgical end points from National Surgical Adjuvant Breast and Bowel Project trial R-04. J Clin Oncol 32: 1927-1934, 2014.

16. Duffy MJ, Lamerz R, Haglund C, Nicolini A, Kalousová M, Holubec L and Sturgeon C: Tumor markers in colorectal cancer, gastric cancer and gastrointestinal stromal cancers: European group on tumor markers 2014 guidelines update. Int J Cancer 134: 2513-2522, 2014

17. Itai S, Fujii Y, Nakamura T, Chang YW, Yanaka M, Saidoh N, Handa S, Suzuki H, Harada H, Yamada S, et al: Establishment of CMab-43, a sensitive and specific anti-CD133 monoclonal antibody, for immunohistochemistry. Monoclon Antib Immunodiagn Immunother 36: 231-235, 2017.

18. Ota T, Suzuki Y, Nishikawa T, Otsuki T, Sugiyama T, Irie R, Wakamatsu A, Hayashi K, Sato H, Nagai K, et al: Complete sequencing and characterization of 21,243 full-length human cDNAs. Nat Genet 36: 40-45, 2004

19. Otsuki T, Ota T, Nishikawa T, Hayashi K, Suzuki Y, Yamamoto J, Wakamatsu A, Kimura K, Sakamoto K, Hatano N, et al: Signal sequence and keyword trap in silico for selection of full-length human cDNAs encoding secretion or membrane proteins from oligo-capped cDNA libraries. DNA Res 12: 117-126, 2005.
20. Kimura K, Wakamatsu A, Suzuki Y, Ota T, Nishikawa T, Yamashita R, Yamamoto J, Sekine M, Tsuritani K, Wakaguri $\mathrm{H}$, et al: Diversification of transcriptional modulation: Large-scale identification and characterization of putative alternative promoters of human genes. Genome Res 16: 55-65, 2006.

21. Itoh M, Yasunishi A, Imamura K, Kanamori-Katayama M, Suzuki H, Suzuki M, Carninci P, Kawai J and Hayashizaki Y: Constructing ORFeome resources with removable termination codons. Biotechniques 41: 44-50, 46, 48 passim, 2006.

22. Dzobo K, Senthebane DA, Ganz C, Thomford NE, Wonkam A and Dandara C: Advances in therapeutic targeting of cancer stem cells within the tumor microenvironment: An updated review. Cells 9: 1896, 2020.

23. O'Brien CA, Pollett A, Gallinger S and Dick JE: A human colon cancer cell capable of initiating tumour growth in immunodeficient mice. Nature 445: 106-110, 2007.

24. Ricci-Vitiani L, Lombardi DG, Pilozzi E, Biffoni M, Todaro M, Peschle $\mathrm{C}$ and De Maria R: Identification and expansion of human colon-cancer-initiating cells. Nature 445: 111-115, 2007.

25. Lugli A, Iezzi G, Hostettler I, Muraro MG, Mele V, Tornillo L, Carafa V, Spagnoli G, Terracciano L and Zlobec I: Prognostic impact of the expression of putative cancer stem cell markers CD133, CD166, CD44s, EpCAM, and ALDH1 in colorectal cancer. Br J Cancer 103: 382-390, 2010.

26. Kato Y, Ohishi T, Yamada S, Itai S, Furusawa Y, Sano M, Nakamura T, Kawada M and Kaneko MK: Anti-CD133 monoclonal antibody CMab-43 exerts antitumor activity in a mouse xenograft model of colon cancer. Monoclon Antib Immunodiagn Immunother 38: 75-78, 2019.

27. Takei J, Kaneko MK, Ohishi T, Kawada M, Harada H and Kato Y: $\mathrm{H}_{2} \mathrm{Mab}-19$, an anti-human epidermal growth factor receptor 2 monoclonal antibody exerts antitumor activity in mouse oral cancer xenografts. Exp Ther Med 20: 846-853, 2020.

28. Kato Y, Ohishi T, Takei J, Nakamura T, Sano M, Asano T, Sayama Y,Hosono H, Kawada M and Kaneko MK: An anti-human epidermal growth factor receptor 2 monoclonal antibody H2Mab-19 exerts antitumor activity in mouse colon cancer xenografts. Monoclon Antib Immunodiagn Immunother 39: 123-128, 2020.

29. Spizzo G, Fong D, Wurm M, Ensinger C, Obrist P, Hofer C, Mazzoleni G, Gastl G and Went P: EpCAM expression in primary tumour tissues and metastases: An immunohistochemical analysis. J Clin Pathol 64: 415-420, 2011.

30. Balzar M, Prins FA, Bakker HA, Fleuren GJ, Warnaar SO and Litvinov SV: The structural analysis of adhesions mediated by Ep-CAM. Exp Cell Res 246: 108-121, 1999.

31. Liao MY, Lai JK, Kuo MY, Lu RM, Lin CW, Cheng PC, Liang KH and Wu HC: An anti-EpCAM antibody EpAb2-6 for the treatment of colon cancer. Oncotarget 6: 24947-24968, 2015.

32. Eyvazi S, Farajnia S, Dastmalchi S, Kanipour F, Zarredar H and Bandehpour M: Antibody Based EpCAM Targeted Therapy of Cancer, Review and Update. Curr Cancer Drug Targets 18: 857-868, 2018.

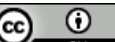

This work is licensed under a Creative Commons Attribution 4.0 International (CC BY 4.0) License. 